\title{
Perioperative Docetaxel, Oxaliplatin, Fluorouracil, and Leucovorin (FLOT) in Patients with Gastric or Esophagogastric Junction Adenocarcinoma; Real-Life Experience
}

\author{
Fatih YILDIZ ${ }^{1}$, Emrah ERASLAN ${ }^{1}$, Aysegul ILHAN ${ }^{1}$, Hacer DEMIR ${ }^{2}$, Nazan DEMIR ${ }^{3}$, \\ Erkan ERDUR ${ }^{4}$, Ozgen Ahmet YILDIRIM ${ }^{4}$, Huseyin KANMAZ ${ }^{5}$, Ferit ASLAN ${ }^{6}$, Gulnihal TUFAN ${ }^{1}$, \\ Ayse DURNALI ${ }^{1}$, Berna OKSUZOGLU ${ }^{1}$, Umut DEMIRCI ${ }^{7}$ \\ ${ }^{1}$ Ankara Oncology Training and Research Hospital, Department of Medical Oncology, Ankara \\ ${ }^{2}$ Afyon Kocatepe University, Faculty of Medicine, Department of Medical Oncology, Afyon \\ ${ }^{3}$ Eskisehir Osmangazi University, Faculty of Medicine, Department of Medical Oncology, Eskişehir \\ ${ }^{4}$ Diyarbakır Gazi Yasargil Training and Research Hospital, Department of Medical Oncology, Diyarbakır \\ ${ }^{5}$ Balıkesir Ataturk City Hospital, Department of Medical Oncology, Balıkesir \\ ${ }^{6}$ Medical Park Hospital, Department of Medical Oncology, Ankara \\ ${ }^{7}$ Uskudar University, Memorial Ankara Hospital, Department of Medical Oncology, Ankara, TURKEY
}

\begin{abstract}
In patients with gastic cancer, five-year survival is poor in the locally advanced stage. Docetaxel, oxaliplatin, leucovorin, and 5-fluorouracil (FLOT) combination regimen has been shown to provide a survival advantage in the locally advanced stage. In this study, we aimed to evaluate the efficacy and tolerability of FLOT with real-life data in patients with locally advanced gastric/esophagogastric junction cancers. This retrospective study was conducted between June 2016 - March 2020 and included 106 patients' data from six centers in Turkey. Median age was 60 (33-82). Primary tumor localization was stomach in 76 (71.7\%) patients. Seventy-six (71.7\%) patients were operated after median 4 (1-8 cycles) cycles of preoperative FLOT. Pathological complete regression (pCR)was obtained in $10(13.1 \%)$ of the operated patients. Median follow-up was 9.1 (1.4-45.7) months. One-year DFS was $63.2 \%$ and the two-year OS was $65.1 \%$. Three (2.8\%) patients had chemotherapy-related deaths. Due to chemotherapy-related toxicity and intoleration, 19 (17.9\%) patients had dose reduction. The PCR obtained by FLOT appears higher than other regimens. This study is one of the rare multicentric real-life data showing the efficacy and tolerability of the FLOT regimen in the perioperative treatment in GC and EJC.
\end{abstract}

Keywords: Gastric cancer, Perioperative chemotherapy, Neoadjuvant chemotherapy, FLOT, Locally advanced gastric cancer

\section{INTRODUCTION}

Gastric cancer (GC) is the fifth most common cancer in the world and is the third most common cause of cancer-related deaths. ${ }^{1}$ The recommended curative treatment for GC is R0 resection and D2 lymph node dissection. However, more than half of the patients are at locally advanced stage and are not suitable for upfront surgery. ${ }^{2-5}$ Five-year overall survival (OS) rates after R0 resection in Stage I GC are around $80 \%$, whereas it declines to $20 \%$ in stage III patients. ${ }^{6-10}$ This is the basis for neoadjuvant therapies especially in the locally advanced stage for the treatment of GC. 
Firstly, in the MAGIC study, 13\% improvement in five-year OS was achieved with perioperative epirubicin, cisplatin, and 5-fluorouracil (ECF) combination chemotherapy in patients with GC in the resectable stage. ${ }^{11}$ In the subsequent French phase 3 study, a similar benefit was obtained with cisplatin, 5-fluorouracil (CF) combination chemotherapy. ${ }^{12}$ In the MRC OE05 trial which compares the triplet cisplatin, epirubicin and capecitabin or 5- fluorouracil (ECX/ECF) regimen with CF regimen, it was observed that the triplet regimen did not increase survival in esophagogastric junction cancers (EJC). ${ }^{13}$

Failure to obtain sufficient benefit with chemotherapy regimens in the preoperative treatment of GC and EJC cancer has directed the researchers to new combinations. In phase $2 / 3$ AIO study published in 2016, preoperative docetaxel, oxaliplatin, 5-Fluorouracil (FLOT) combination regimen was compared with ECX in resectable GC and EJC. ${ }^{14}$ As a result of this study, it was observed that a higher rate of complete response $(\mathrm{CR})$ was obtained with the FLOT regimen. In the phase $2 / 3$ results published in 2019 of the same study, it was observed that the FLOT regimen was superior in terms of OS. ${ }^{15}$

To the best of our knowledge, there are no major studies on real-life data other than the phase $2 / 3$ study on the efficacy and toxicity of the FLOT regimen in perioperative treatment. In this study, we aimed to evaluate the efficiency and tolerability of the perioperative FLOT regimen in the treatment of locally advanced gastric and esophagogastric junction carcinomas with real-life data retrospectively.

\section{PATIENTS and METHODS}

This study was performed between June 2016March 2020 in six different centers in Turkey. Patients older than 18 years of age and who received perioperative FLOT regimen for locally advanced GC/EJC were included in the study. Ethics committee approval was obtained before the study.

Demographic and clinicopathological characteristics of the patients, preoperative American Joint Committee on Cancer (AJCC) TNM stage, postoperative pathological stage, pathological regression grade, chemotherapy-related toxicity, progression, and mortality status were recorded retrospectively through electronic registry system and manual patient files.

Tumor regression grade (TRG) was evaluated according to Becker Regression Criteria. ${ }^{16}$ The factors that may be associated with the pathological response were analyzed in the patients who had surgery. Chemotherapy-related toxicities were graded according to Common Terminology Criteria for Adverse Events version 4.03. ${ }^{17}$ All patients in the study were included in the toxicity assessment. Response Evaluation Criteria In Solid Tumors (RECIST) version 1.1 was used for radiological response evaluation. ${ }^{18}$

The data were evaluated by the IBM Statistical Package for Social Sciences (SPSS ${ }^{\circledR}$ ) v.21 (IBM Inc.; Armonk, NY, USA). The time from the diagnosis to the death was defined as overall survival (OS), and the time from the surgery to the recurrence was defined as disease free survival (DFS). Survival analyzes were performed with Kaplan-Meier method. The relationship between categorical variables and the pathological response was evaluated by chi-square test. Receiver operator characteristic (ROC) analysis was used to determine the optimum cut-off value of neutrophil/ lymphocyte ratio (NLR) in terms of pathological response.

\section{RESULTS}

The median age of the 106 patients who were included in the study was 60 (33-82) and $77.4 \%$ were male $(n=82)$. Primary tumor localization was stomach in $76(71.7 \%)$ patients. Patient characteristics are shown in Table 1. Almost all patients ( $\mathrm{n}=$ $103,97.2 \%$ ) were clinically lymph node positive at the time of diagnosis.

FLOT was given once every two-week as docetaxel $50 \mathrm{mg} / \mathrm{m}^{2}$ intravenous on day 1 , oxaliplatin 85 $\mathrm{mg} / \mathrm{m}^{2}$ intravenous on day 1 , leucovorin $200 \mathrm{mg} /$ $\mathrm{m}^{2}$ intravenous on day 1 , and 5-fluorouracil 2600 $\mathrm{mg} / \mathrm{m}^{2}$ as $48 \mathrm{~h}$ infusion on day 1-2. Primary granulocyte colony stimulating factor (G-CSF) prophylaxis was applied to $87(82.1 \%)$ patients and secondary G-CSF prophylaxis to $12(11.3 \%)$ patients. 


\begin{tabular}{|c|c|c|}
\hline & $n=106$ & $\%$ \\
\hline \multicolumn{3}{|l|}{ Age } \\
\hline Median (range) - yr & $60(33-82)$ & \\
\hline \multicolumn{3}{|l|}{ Sex } \\
\hline Male & 82 & 77.4 \\
\hline Female & 24 & 22.6 \\
\hline \multicolumn{3}{|l|}{ ECOG performance status score } \\
\hline 0 or 1 & 105 & 99.1 \\
\hline 2 & 1 & 0.9 \\
\hline \multicolumn{3}{|l|}{ Histology } \\
\hline Adenocarcinoma & 94 & 88.6 \\
\hline Signet-ring cell carcinoma & 12 & 11.3 \\
\hline \multicolumn{3}{|l|}{ Primary tumour location } \\
\hline Gastric & 76 & 71.7 \\
\hline Gastro-oesophageal junction & 30 & 28.3 \\
\hline \multicolumn{3}{|l|}{ Clinical tumour stage } \\
\hline cT2/cT3 & 96 & 90.5 \\
\hline cT4 & 10 & 9.4 \\
\hline \multicolumn{3}{|l|}{ Clinical node stage } \\
\hline $\mathrm{cN}+$ & 103 & 97.2 \\
\hline $\mathrm{cN}-$ & 3 & 2.8 \\
\hline
\end{tabular}

Seventy-six $(71.7 \%)$ patients were operated after median 4 (1-8) cycles of preoperative FLOT. R0 resection was performed in $72(94.7 \%)$ of these patients and R1 resection in $4(5.2 \%)$ patients. Twenty-one $(19.8 \%)$ patients did not have resectional surgery. Reasons for not proceeding to surgery were clinical or radiological progression of disease in $9(8.4 \%)$ patients, refused the treatment in $7(6.6 \%)$ patients, worsening in performance status due to chemotherapy-related toxicity in $2(1.8 \%)$ patients, chemotherapy-related death in $3(2.8 \%)$ patients. Preoperative FLOT was still ongoing in 9 $(8.4 \%)$ patients.

Pathological complete regression (pCR) was obtained in $13.1 \%(n=10)$ of the operated patients. This ratio was $13.8 \%$ for the patients who treated with at least four cycles FLOT regimen. The proportion of TRG 1 patients were $31.5 \%(n=24)$. TRG was 2 (10\%-50\% residual tumor/tumor bed) in $28(37.3 \%)$ patients, TRG was 3 (> 50\% residual tumor/tumor bed) in $23(30.6 \%)$ patients (Table 2 ). When the factors that may be associated with

\begin{tabular}{|lll|}
\hline \multicolumn{2}{|l|}{ Table 2. Pathological results } & \\
\hline & $\mathbf{n}=\mathbf{7 6}$ & $\%$ \\
\hline Pathological tumour stage (ypT) & & \\
$\quad$ ypT0 & 10 & 13.1 \\
ypT1 & 6 & 7.8 \\
ypT2 & 5 & 6.5 \\
ypT3 & 43 & 56.5 \\
ypT4 & 12 & 15.7 \\
Pathological nodal stage (ypN) & & \\
ypN0 & 32 & 42.1 \\
ypN1 & 15 & 19.7 \\
ypN2 & 15 & 19.7 \\
ypN3 & 14 & 18.4 \\
Histopathological tumour regression & & \\
Complete (TRG* 1a) & 10 & 13.1 \\
Subtotal (TRG 1b) & 14 & 18.4 \\
Partial (TRG 2) & 29 & 38.1 \\
Minimal or none (TRG 3) & 23 & 30.2 \\
& & \\
\hline * TRG= tumour regression grade & & \\
& & \\
\hline
\end{tabular}

the pathological response were analyzed, it was observed that the primary tumor localization, clinical $\mathrm{T}$ stage, neutrophil /lymphocyte ratio was not related to the tumor response (Table 3).

FLOT protocol was continued in $52(49.0 \%)$ patients as a postoperative treatment. Chemoradiotherapy (with capecitabine) in $10(9.4 \%)$ patients and capecitabine plus oxaliplatin in 4 (3.7\%) patients preferred as an adjuvant treatment. Ten (9.4\%) patients did not start postoperative treatment. Reasons for not starting postoperative treatment were postoperative complications in $5(4.7 \%)$ patients, death due to surgical complications in 1 $(0.09 \%)$ patient, and refusing treatment in $4(3.7 \%)$ patients.

Forty-two (39.6\%) patients completed the perioperative eight cycles FLOT. Median follow-up was 9.1 (1.4-45.7) months. Distant metastases were confirmed in 10 (9.4\%) patients. One-year DFS was $63.2 \%$ (Figure 1). Seventeen $(16.0 \%$ ) patients had died and the two-year OS was $65.1 \%$ (Figure 2). 


\begin{tabular}{|c|c|c|c|}
\hline & $\begin{array}{l}\text { Tumour regression } \\
\text { grade } 1 \text { (n) }\end{array}$ & $\begin{array}{l}\text { Tumour regression } \\
\text { grade } 2-3(n)\end{array}$ & p-value \\
\hline \multicolumn{4}{|l|}{ Sex } \\
\hline Male & 40 & 16 & 0.78 \\
\hline Female & 12 & 8 & \\
\hline \multicolumn{4}{|l|}{ Primary tumour location } \\
\hline Gastro-oesophageal junction & 10 & 9 & 0.06 \\
\hline Gastric & 43 & 14 & \\
\hline \multicolumn{4}{|l|}{ Histology } \\
\hline Adenocarcinoma & 44 & 21 & 0.48 \\
\hline Signet-ring cell carcinoma & 9 & 2 & \\
\hline \multicolumn{4}{|l|}{ Neutrophil/lymphocyte ratio } \\
\hline$<5$ & 44 & 22 & 0.25 \\
\hline$>5$ & 7 & 3 & \\
\hline \multicolumn{4}{|l|}{ Clinical tumour stage } \\
\hline сT2/сT3 & 41 & 27 & 0.49 \\
\hline cT4 & 5 & 3 & \\
\hline
\end{tabular}

When the toxicities were evaluated, $3(2.8 \%)$ patients had chemotherapy-related deaths. Due to chemotherapy-related toxicity and intoleration, 19 $(17.9 \%)$ patients had dose reduction. Chemotherapy delay occurred in $50(47.1 \%)$ patients. The most common side effect was nause $(n=76,71.6 \%)$. Grade 3-4 neutropenia occurred in 30 (28.3\%) patients, while FN was in seven $(6.6 \%)$ patients. Chemotherapy related toxicities are shown in Table 4 .

\section{DISCUSSION}

To the best of our knowledge, this study is the largest real-life data that evaluates the efficacy and safety of FLOT in perioperative treatment in GC and EJC except phase 2/3 study. In our study, pCR was obtained in $13 \%$ of patients with FLOT regimen.

The short survival times in locally advanced GC and EJC cancer has directed the researchers to preoperative chemotherapy approaches. The first major study on this subject is the MAGIC study published in 2006. In this study, the perioperative ECF regimen was compared with upfront surgery in 503 operable patients. The majority (74\%) of patients were GC. In this study, the five-year OS was found to be better in the ECF arm than the surgical arm (36\% vs 23\%). The pathological complete response (pCR) ratio has not been reported. ${ }^{11}$

A total of 144 patients with GC and EJC were included in the randomized study of the European Organization for Research and Treatment of Cancer (EORTC) in 2010. Patients were randomized to the preoperative $\mathrm{CF}$ and upfront surgery arms. ${ }^{19}$ Although the $\mathrm{R} 0$ resection rate is higher in patients receiving neoadjuvant therapy, it has been reported that this does not provide survival benefit. ${ }^{19}$ In the phase 3 French study 224 patients (64\% EJC, 25\% GC, $11 \%$ esophagus carcinomas) were randomized to upfront surgery and neoadjuvant CF arms. ${ }^{12}$ In this study the $\mathrm{pCR}$ rate was not reported, however, five-year OS was better in the CF arm than the upfront surgery arm (38\% vs 24\%). Five-year DFS was $34 \%$ in $\mathrm{CF}$ arm. Although these three studies are accepted as landmark studies showing the effectiveness of neoadjuvant therapy in GC and EJC, the pathological response rate has not been reported.

The one-year DFS obtained in our study was $63.2 \%$ and the two-year OS was $65.1 \%$. In almost all of the studies in the literature five-year OS and DFS are mentioned. Therefore, we could not compare the one-year DFS and two-year OS results, 


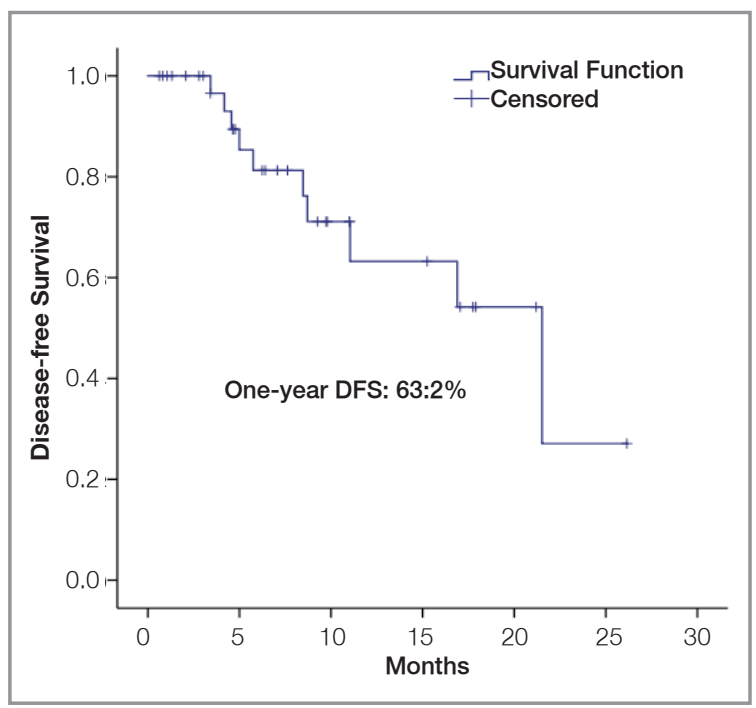

Figure 1. Kaplan-Meier estimates of disease-free survival

which obtained in our study, with other studies. It will be more accurate to compare with other studies in terms of OS and DFS after a longer followup period.

In a small, single-center phase 2 study, 34 patients with esophageal/EJC achieved $4 \%$ pCR with 4 cycles of neoadjuvant ECX. ${ }^{20}$ In phase 3 OE05 study, neoadjuvant CF and ECX were compared in 897 esophageal adenocarcinomas. pCR proportion has been reported as $7 \%$ vs $2 \%$, respectively. ${ }^{13}$ In another multi-center phase $2 / 3$ study performed in the $\mathrm{UK}$, it was seen that $5 \%$ pCR was obtained with neoadjuvant ECX. ${ }^{21}$

While the pCR obtained with preoperative doublet/ triplet treatments did not exceed $7 \%$ in all the studies mentioned, this rate increased to $16 \%$ with the FLOT protocol in the AIO study. In the phase 2/3 FLOT4-AIO study published in 2016, 265 patients with GC or EJC were randomized to $\mathrm{ECF} / \mathrm{ECX}$ and FLOT arms and the pathological response rates were compared after neoadjuvant therapy. ${ }^{14} \mathrm{GC}$ and EJC were almost equal in this study, whereas pCR was $16 \%$ in the FLOT group and $6 \%$ in the $\mathrm{ECF} / \mathrm{ECX}$ arm. In our study which reflects the real-life experience, $\mathrm{pCR}$ was $13.8 \%$ for the patients who treated with at least four cycles FLOT regimen. It was similar to phase $2 / 3$ study. While the proportion of the patients with pathological total or subtotal regression (TRG1) was $37 \%$ in AIO, it was $31.5 \%$ in our study. Based on this, we can say

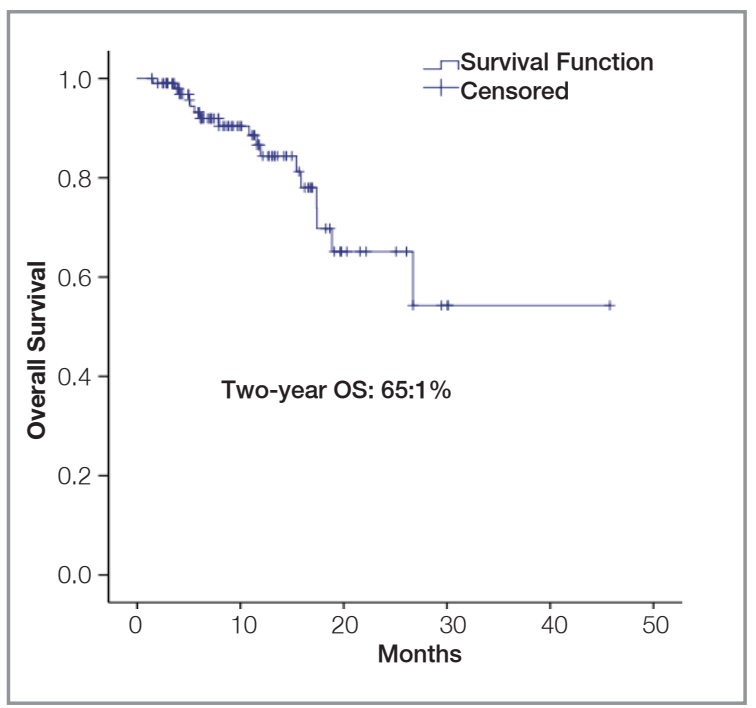

Figure 2. Kaplan-Meier estimates of overall survival

that the efficacy results with neoadjuvant FLOT of our real-life data are similar to those in phase $2 / 3$ study.

When the toxicity of combination regimens used in the preoperative treatment of GC and EJC are evaluated, $20 \%-52 \%$ grade 3-4 neutropenia is observed in both doublet (CF) and triplet (ECF, ECX, FLOT) regimens. ${ }^{11-13,20}$ Neutropenia has been reported most frequently in patients treated with FLOT. Grade 3-4 neutropenia with FLOT developed in $52 \%$ of the patients in the AIO study and $28.3 \%$ in our study. ${ }^{14}$ Primary G-CSF prophylaxis was applied to $82.1 \%$ of the patients in our study, resulting in a lower rate of neutropenia. In our daily practice, the low socio-cultural level of our patients, and the fact that they live in other cities make us more liberal in the use of G-CSF for primary prophylaxis. However, FN was $2 \%$ with FLOT in the phase $2 / 3$ pivotal study and $6.6 \%$ in our study. ${ }^{15}$ Although we used more primary GCSF prophylaxis, the FN rate was slightly higher in our study. This may be due to the low sociocultural level of our patients and lack of self-care.

Grade 3-4 chemotherapy-related nausea/vomiting is similar in FLOT and other perioperative regimens. While grade 3-4 nausea/vomiting develops with other doublet and triplet treatments in the $5-9 \%$ of the patients, it is approximately $9 \%$ with FLOT. ${ }^{11,12,14,19}$ Similarly, in our study, $9.4 \%$ of patients had grade 3-4 nausea. Grade 3-4 diarrhea oc- 
Table 4. Chemotherapy related toxicity $(n=106)$

\begin{tabular}{|c|c|c|c|}
\hline & N/A & Grade 1 or 2 & Grade 3 or more \\
\hline \multicolumn{4}{|l|}{ Hematological } \\
\hline Anemia & & 73 (\%68.8) & $2(1.8 \%)$ \\
\hline Thrombocytopenia & & $15(14.1 \%)$ & $3(2.8 \%)$ \\
\hline Neutopenia & & 18 (16.9\%) & 30 (28.3\%) \\
\hline Febrile neutropenia & & - & 7 (6.6\%) \\
\hline \multicolumn{4}{|l|}{ Non-hematological } \\
\hline Fatigue & & 65 (61.3\%) & 10 (9.4\%) \\
\hline Nausea & & $66(62.2 \%)$ & $10(9.4 \%)$ \\
\hline Vomiting & & $28(26.4 \%)$ & $2(1.8 \%)$ \\
\hline Diarrhea & & $15(14.1 \%)$ & $4(3.7 \%)$ \\
\hline Chemotherapy delay & $50(47.1 \%)$ & & \\
\hline Dose reduction & 19 (17.9\%) & & \\
\hline Chemotherapy related death & $3(2.8 \%)$ & & \\
\hline
\end{tabular}

curs less than $3 \%$ with other regimens, while it is reported in $10 \%$ of patients under the FLOT regimen. In our study, diarrhea was seen relatively few.

In the AIO study, $18 \%$ of patients had dose reduction in the preoperative period and $21 \%$ in the postoperative period. ${ }^{14} \mathrm{We}$ observed that $17.9 \%$ of the patients received dose modification. In our study, the number of patients with dose reduction appeared less. A high rate of primary G-CSF prophylaxis may have decreased the dose reduction caused by myelosuppression. At the same time, it should not be forgotten that there are still patients having ongoing treatment.

The limitations of our study are its retrospective nature, short follow-up time and the low number of patients. In retrospective observational studies, toxicity records may be underreported. The advantage of having a multicentric study is that it allows for a larger and different patient population. The disadvantage is that the pathological evaluation is not done from a single center.

In conclusion, our study is one of the rare multicentric real-life data showing the efficacy and tolerability of the FLOT regimen in the perioperative treatment in GC and EJC. The data we obtained are parallel to the pivotal study. The $\mathrm{pCR}$ obtained by FLOT appears higher than other regimens. Perioperative treatment decision in this patient group should be made in multidisciplinary councils.

\section{REFERENCES}

1. Bray F, Ferlay J, Soerjomataram I, et al. Global cancer statistics 2018: GLOBOCAN estimates of incidence and mortality worldwide for 36 cancers in 185 countries. CA Cancer J. Clin 68: 394-424, 2018.

2. Smith DD; Schwarz RR, Schwarz RE. Impact of total lymph node count on staging and survival after gastrectomy for gastric cancer: Data from a Large US-Population Database. J Clin Oncol 23: 7114-7124, 2005.

3. Hartgrink $\mathrm{HH}$, van de Velde $\mathrm{CJ}$, Putter $\mathrm{H}$, et al. Extended lymph node dissection for gastric cancer: who may benefit? Final results of the randomized Dutch gastric cancer group trial. J Clin Oncol 22: 2069-2077, 2004.

4. Bonenkamp JJ, Hermans J, Sasako M, et al. Extended lymph-node dissection for gastric cancer. N Engl J Med 340: 908-914 1999.

5. Cuschieri A, Fayers P, Fielding J, et al. Postoperative morbidity and mortality after D1 and D2 resections for gastric cancer: preliminary results of the MRC randomised controlled surgical trial. The Surgical Cooperative Group. Lancet 347: 995-999, 1996.

6. Reim D, Loos M, Vogl F, et al. Prognostic implications of the seventh edition of the International Union Against Cancer classifi cation for patients with gastric cancer: the Western experience of patients treated in a single-center European institution. J Clin Oncol 3: 263-271, 2013.

7. Davis PA, Sano T. The difference in gastric cancer between Japan, USA and Europe: what are the facts? what are the suggestions? Crit Rev Oncol Hematol 40: 77-94, 2001.

8. Gill S, Shah A, Le N, et al. Asian ethnicity-related differences in gastric cancer presentation and outcome among patients treated at a canadian cancer center. J Clin Oncol 21: 20702076, 2003. 
9. Strong VE, Song KY, Park $\mathrm{CH}$, et al. Comparison of gastric cancer survival following $\mathrm{RO}$ resection in the United States and Korea using an internationally validated nomogram. Ann Surg 25: 640-646, 2010.

10. Wanebo HJ, Kennedy BJ, Chmiel J, et al. Cancer of the stomach. A patient care study by the American College of Surgeons. Ann Surg 218: 583-592, 1993.

11. Cunningham D, Allum WH, Stenning SP, et al. Perioperative chemotherapy versus surgery alone for resectable gastroesophageal cancer. N Engl J Med 355: 11-20, 2006.

12. Ychou M, Boige V, Pignon JP, et al. Perioperative chemotherapy compared with surgery alone for resectable gastroesophageal adenocarcinoma: An FNCLCC and FFCD multicenter phase III trial. J Clin Oncol 29: 1715-1721, 2011.

13. Alderson D, Cunningham D, Nankivell M, et al. Neoadjuvant cisplatin and fluorouracil versus epirubicin, cisplatin,and capecitabine followed by resection in patients with oesophagealadenocarcinoma (UK MRC OEO5): An open-label, randomised phase 3 trial. Lancet Oncol 18: 1249-1260, 2017.

14. Al-Batran SE, Hofheinz RD, Pauligk C, et al. Histopathological regression after neoadjuvant docetaxel, oxaliplatin, fluorouracil, and leucovorin versus epirubicin, cisplatin, and fluorouracil or capecitabine in patients with resectable gastric or gastro-oesophageal junction adenocarcinoma (FLOT4-AIO): results from the phase 2 part of a multicentre, open-label, randomised phase 2/3 trial. Lancet Oncol 17: 1697-708, 2016.

15. Al-Batran SE, Homann N, Pauligk C, et al. Perioperative chemotherapy with fluorouracil plus leucovorin, oxaliplatin, and docetaxel versus fluorouracil or capecitabine plus cisplatin and epirubicin for locally advanced, resectable gastric or gastro-oesophageal junction adenocarcinoma (FLOT4): a randomised, phase 2/3 trial. Lancet 393: 1948-1957, 2019.

16. Becker K, Mueller JD, Schulmacher C, et al. Histomorphology and grading of regression in gastric carcinoma treated with neoadjuvant chemotherapy. Cancer 98: 1521-1530, 2003.

17. https://ctep.cancer.gov/protocoldevelopment/electronic_applications/docs/CTCAE_v5_Quick_Reference_8.5x11.pdf (Accessed on March 09, 2018).

18. Eisenhauer EA, Therasse P, Bogaerts J, et al. New response evaluation criteria in solid tumours: revised RECIST guideline (version 1.1). Eur J Cancer 45: 228-247, 2009.

19. Schuhmacher C, Gretschel S, Lordick F, et al. Neoadjuvant chemotherapy compared with surgery alone for locally advanced cancer of the stomach and cardia: European Organisation for Research and Treatment of Cancer randomized trial 40954. J Clin Oncol 28: 5210-5218, 2010.
20. Starling N, Okines A, Cunningham D, et al. A phase II tria of preoperative chemotherapy with epirubicin, cisplatin and capecitabine for patients with localised gastro-oesophageal junctional adenocarcinoma. Br J Cancer 100: 1725-1730, 2009.

21. Cunningham D, Stenning SP, Smyth EC, et al. Peri-operative chemotherapy \pm bevacizumab for resectable gastro-oesophageal adenocarcinoma: results from the UK Medical Research Council randomised ST03 trial (ISRCTN 46020948). Eur J Cancer 51 Suppl 3: 400, 2015.

\section{Correspondence: \\ Dr. Fatih YILDIZ}

Dr. Abdurrahman Yurtaslan Ankara Onkoloji Hastanesi

M. Akif Ersoy Mahallesi 13. Cadde No: 56

06200 Yenimahalle, ANKARA / TURKEY

Tel: $\quad(+90-506) 3665181$

Fax: (+90-312) 3360909

e-mail: dr.fatihyildiz@hotmail.com

\section{ORCIDs:}

Fatih Yildiz

0000-0003-2295-7332

Emrah Eraslan

0000-0003-2497-5913

Aysegul Ilhan

0000-0002-0333-1388

Hacer Demir

0000-0003-1235-9363

Nazan Demir

0000-0002-2177-7260

Erkan Erdur

0000-0002-9123-2688

Ozgen Ahmet Yildirim

0000-0002-4139-067X

Huseyin Kanmaz

0000-0002-0530-1596

Ferit Aslan

0000-0002-9153-6921

Gülnihal Tufan

0000-0003-2492-3937

Ayse Durnali

Berna Oksuzoglu

0000-0001-9519-6373

Umut Demirci 